\section{Therapeutische Effekte von Qigong bei COPD in der stabilen Phase}

\author{
Originalpublikation
}

Tong H, Liu Y, Zhu Y, Zhang B, Hu J: The therapeutic effects of qigong in patients with chronic obstructive pulmonary disease in the stable stage: a meta-analysis. BMC Complementary and Alternative Medicine. 2019; 19: 239

Der Beitrag ist frei im Internet verfügbar unter https: / /bmccomplementaltern med.biomedcentral.com/articles/10.1186 /s12906-019-2639-9

Teile des Beitrags aus dem Englischen übersetzt bzw. leicht modifiziert durch S. Peters; statistische Kennwerte und Referenzen zum Zwecke der Lesbarkeit entfernt

\section{Ziele}

Die chronisch obstruktive Lungenerkrankung (COPD) ist eine globale Erkrankung. Die Lungenfunktion nimmt kontinuierlich ab. Medikation bildet die Einschränkung des Atemstroms nicht vollständig zurück. Die Rolle von Qigong in der Rehabilitation von COPD wurde ausgewertet. Die vorliegende Metaanalyse hatte zum Ziel, die Effekte von Qigong, durchgeführt von Personen mit COPD, zu bewerten.

\section{Methoden}

Geeignete Studien wurden über eine systematische Suche bezogen. Die Datenbanken wurden am 8. Oktober 2017 durchsucht. Eine Einschränkung bzgl. des Publikationszeitraums wurde nicht gewählt. Um die Qualität geeigneter Studien zu evaluieren, wurde das Cochrane risk-of-bias Tool eingesetzt. Um die Ergebnisse zu analysieren, wurden Mittelwertdifferenzen mit 95\% Konfidenzintervallen verwendet.

\section{Ergebnisse}

10 eingeschlossene Studien umfassten 993 Teilnehmer. Statistische Verbesserungen traten auf bei der 6-Minuten-Gehstrecke (6MWD), dem forcierten ExpirationsSekundenvolumen $\left(\mathrm{FEV}_{1}\right)$, der forcierten Vitalkapazitätsrate von einer Sekunde
$\left(\mathrm{FEV}_{1} / \mathrm{FVC}\right)$, dem forcierten ExpirationsSekundenvolumen/prädiziert $\quad\left(\mathrm{FEV}_{1} /\right.$ pre), einer „Monitored Functional Task Evaluation" (Anm.: Aktivitäten des täglichen Lebens), einem COPD Assessment Test für körperliches Training und dem SF-36 Lebensqualitätsfragebogen (general health und mental health).

\section{Diskussion}

Die Rehabilitation ist eine zentrale Behandlung für COPD-Patienten, empfohlen durch internationale und nationale Leitlinien. Angeratene Bausteine umfassen körperliches Training, patientenorientierte Edukation und Rauchverzicht. Als wichtige Ergänzung des körperlichen Trainings von COPD-Patienten erreicht Qigong körperliche und mentale Integration durch spezielle Handlungen, Atemtechniken und Meditation, welche die Energie des Patienten (Qi) regulieren können, um der körperlichen, psychischen und spirituellen Gesundheit zu nützen. Diese Metaanalyse wurde durchgeführt, um die kurativen Effekte von Qigong (z. B. Baduanjin, Yijinjing, Liuzijue und reproduziertem Qigong) zu evaluieren bei Patienten mit stabiler COPD. Hier hat man sich nur auf Qigong bezogen, was sich von früheren Forschungsarbeiten unterscheidet, die die Wirksamkeit von Tai Chi und Qigong zur selben Zeit untersuchten oder unterschiedliche Stile nicht in Subgruppen unterteilten in ihren Analysen. In der vorliegenden Analyse waren Subgruppen gebildet worden, basierend auf den verschiedenen Qigong-Stilen, um sowohl den Gesamteffekt zu überprüfen als auch den Effekt jedes Stils, was COPD-Patienten eine bessere Möglichkeit zur informierten Auswahl bieten könnte, wenn diese mit so vielen Trainingsformen konfrontiert werden. Generell fand die Studie heraus, dass Qigong-Übungsformen im Vergleich mit konventioneller Behandlung die Rehabilitation bei stabiler COPD unterstützen könnte, bewertet anhand von $6 \mathrm{MWD}, \mathrm{FEV}_{1}, \mathrm{FEV}_{1}$ / FVC, $\mathrm{FEV}_{1} /$ pre, Functional Task Evaluation, SF-36 (general health) und COPD Assessment Test für körperliches Training. Beim SF-36, mental health, gab es keine signifikante Verbesserung. (...)

Die Ergebnisse der Subgruppenanalyse deuten an, dass Baduanjin und Yijinjing signifikant die Lungenfunktion verbessern könnten, während Studien mit dem Fokus auf Liuzijue und eine reproduzierte Form von Qigong keine signifikante Verbesserung der Lungenfunktion fanden. Das könnte teilweise daran liegen, weil Baduanjin und Yijinjing die Atemmuskulatur stärken, das Residualvolumen reduzieren, die Effizienz beim Gasaustausch unterstützen und die Verringerung der Lungenfunktion verlangsamen können. Hinsichtlich Liuzijue zeigten sich in der einen klinischen Studie keine signifikanten Verbesserungen bezüglich dieser Funktionen, vielleicht weil im Vergleich zu Baduanjin und Yijinjing nicht ausreichend Bewegung der oberen Extremitäten stattfindet. Hinsichtlich des reproduzierten Qigongs könnte die Kontinuität der Bewegung verloren gehen, wenn Bewegungsformen aus Yijinjing, Wuqinxi, Liuzijue und Baduanjin ausgewählt werden. Trotzdem könnten diese Praktiken die Immunfunktion verbessern und einen besseren protektiven Effekt gegen akute Exazerbation haben, was für COPD-Patienten von Vorteil wäre.

AKTUELLES ZUM WEITERLESEN (FREI VERFÜGBAR; BEITRÄGE AUF ENGLISCH)

Geidl et al. (2019): Körperliche Aktivität und sedentäre Verhaltensmuster von 326 Personen mit COPD vor dem Start einer pulmonalen Rehabilitation: https: / www.ncbi.nlm.nih.gov/ pmc/articles/PMC6780222/

Janssens et al. (2019): Der Einfluss von krankheitsspezifischen Ängsten (Anm.: inkl. Bewegungsangst) auf die Verläufe von pulmonaler Rehabilitation bei COPD Patienten: https: / /w ww.ncbi.nlm.nih.gov/pmc/articles/ PMC6780973/

\section{Autorinnen / Autoren}

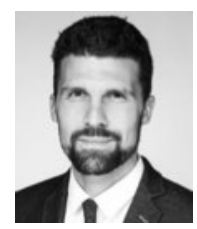

\section{Stefan Peters}

Deutscher Verband für Gesundheitssport und Sporttherapie (DVGS) e. V. Vogelsanger Weg 48 50354 Hürth-Efferen E-Mail: stefan.peters@dvgs.de 